\title{
En bloc procurement of porcine abdominal multiple organ block for ex situ normothermic machine perfusion: a technique for avoiding initial cold preservation
}

\author{
Chuanbao Chen ${ }^{1,2,3 \#}$, Maogen Chen ${ }^{1,2,3}$, Xiaohong Lin ${ }^{4 \#}$, Yiwen Guo ${ }^{1,2,3}$, Yihao Ma ${ }^{1,2,3}$, Zhitao Chen ${ }^{1,2,3}$, \\ Weiqiang $\mathrm{Ju}^{1,2,3}$, Xiaoshun $\mathrm{He}^{1,2,3}$ \\ ${ }^{1}$ Organ Transplant Center, First Affiliated Hospital of Sun Yat-sen University, Guangzhou, China; ${ }^{2}$ Guangdong Provincial Key Laboratory of Organ \\ Donation and Transplant Immunology, Guangzhou, China; ${ }^{3}$ Guangdong Provincial International Cooperation Base of Science and Technology \\ (Organ Transplantation), Guangzhou, China; ${ }^{4}$ Division of General Surgery, The Eastern Hospital of the First Affiliated Hospital of Sun Yat-sen \\ University, Guangzhou, China \\ Contributions: (I) Conception and design: C Chen, M Chen, X Lin; (II) Administrative support: X He, W Ju; (III) Provision of study materials or \\ patients: M Chen, X He, W Ju; (IV) Collection and assembly of data: Y Ma, Y Guo, Z Chen; (V) Data analysis and interpretation: C Chen, M Chen, \\ X Lin; (VI) Manuscript writing: All authors; (VII) Final approval of manuscript: All authors. \\ \#These authors contributed equally to this work. \\ Correspondence to: Xiaoshun He; Weiqiang Ju. Organ Transplant Center, First Affiliated Hospital of Sun Yat-sen University, No. 58 Zhongshan 2nd \\ Road, Guangzhou 510080, China. Email: hexsh@mail.sysu.edu.cn; weiqiangju@163.com.
}

Background: Normothermic machine perfusion (NMP) is a technique that maintains organs ex situ with normal metabolism, and organ function can be better preserved. The study of multiple-organ NMP is rarely reported. Multiple organ block (MOB) is a self-perfusing system for maintaining multiple organs ex situ, and porcine MOBs have been successfully preserved for 18 to $37 \mathrm{~h}$. Due to the above context, we conceived to maintain abdominal multiple organ block (AMOB) ex situ utilizing NMP technology.

Methods: AMOBs were procured from Ba-Ma miniature pigs through en bloc procurement surgery. The process of cold preservation was eliminated between the procurement and machine perfusion, and a few minutes of warm ischemia emerged. Autologous whole blood was collected during procurement surgery as a perfusate component in the beginning.

Results: The median time of procurement surgery was approximately $220 \mathrm{~min}$, and the median time of warm ischemia was $300 \mathrm{sec}$. Cases 1 and 2 suffered from repeated hypotension during the procurement surgery, and case 2 exhibited hemorrhage. After improved and optimized procurement processes, the vital signs of cases 3 to 5 remained stable during procurement. In the NMP phase, the flow increased slowly in cases 1 and 2 and did not remain stable even after continuous infusion of a high-dose vasodilator. The lactic acid level rapidly increased, and the levels of ALT and AST were obviously higher than those in cases 3 to 5. In contrast, the flow rate increased smoothly in cases 3 to 5 . The lactic acid level remained stable during the first $10 \mathrm{~h}$ of perfusion.

Conclusions: AMOB procurement from heart-beating pigs for NMP without initial cold preservation is technically feasible.

Keywords: Normothermic machine perfusion (NMP); abdominal multiple organ block (AMOB); en bloc procurement; organ preservation

Submitted Mar 19, 2021. Accepted for publication Jun 11, 2021.

doi: 10.21037/atm-21-1308

View this article at: https://dx.doi.org/10.21037/atm-21-1308 


\section{Introduction}

Organ transplantation is the optimal choice to prolong the lives of people with chronic end-stage disease. Organ preservation is a key component of successful organ transplantation, and the development of organ preservation techniques has greatly promoted organ transplantation (1-3). There are two main organ preservation methods distinguished by temperature: cold preservation and warm preservation.

Cold preservation decreases organ temperature, reduces enzyme activity and slows cell metabolism, hence prolonging the preservation time of isolated organs (4). With the advantages of a simple technique, low cost and ease of transport, cold preservation has become a widely used technology in the clinic, and static cold storage (SCS) with the University of Wisconsin (UW) solution is the gold standard in organ preservation (5).

Hypothermic machine perfusion (HMP) is another type of cold preservation. Compared to SCS, HMP provides continuous perfusion with cold perfusate via a perfusion machine, can remove metabolic waste in a timely manner and provides some metabolic substrates. HMP can better protect organ function and decrease the rate of delayed graft function (DGF) (6,7). Another advantage of HMP is assistance in assessing organ function according to the perfusion parameters (8). Hypothermic oxygenated perfusion (HOPE) is a new technique with an additional oxygen supply based on HMP and has been reported to be excellent for organ preservation (9-11).

In contrast to cold preservation, warm preservation is a technique that ex situ mimics the normal physiological state with the aid of a normothermic machine perfusion (NMP) device. Compared to SCS and HMP, organs can maintain normal metabolism ex vivo through the NMP technique, and the preservation effect of NMP has been proven in many studies. NMP has been shown to sustain the liver ex vivo for 7 days, and liver function remained good (3). Randomized controlled clinical studies have also shown some advantages of NMP over SCS in liver preservation (12). In addition, NMP has a unique advantage in organ function assessment. NMP assists in assessing organ function according to viability criteria, reduces the rate of organ discard and improves the organ pool $(13,14)$.

Ex vivo maintenance of a single organ with NMP is currently the main focus, but few studies have reported the perfusion of multiple organs (15). A self-perfusing system called multiple organ block (MOB), which contains the heart, lungs, kidneys, liver, pancreas, and bowel and is en bloc procured with the vascular system, has been reported. The heart maintains the blood circulation, and the lung provides oxygenation under artificial ventilation (16). MOBs from pigs have been preserved ex situ for 18 to $37 \mathrm{~h}$ with this selfperfusing system (17).

Based on the above research, we conceived to procure abdominal multiple organ block (AMOB) using the surgical method of en bloc procurement with the vascular system. AMOB contains multiple abdominal organs and maintains the normal anatomic relationship. Unlike the self-perfusing system of MOB, AMOBs were transferred to an NMP device for ex situ perfusion of multiple organs.

NMP technology has been widely used in our center $(15,18-20)$, and a new concept of organ-oriented research and treatment were introduced by our team (21). Organoriented research and treatment represents the study or cure of a disease at the organ level with the help of NMP technology in situ or ex situ. The overarching idea is to combine NMP with advances in surgery, pharmacology, and teaching and so on. To maintain AMOBs ex situ by NMP is part of the organ-oriented research and treatment.

Two improvements were documented in this research: elimination of cold preservation and collection of autologous whole blood as a perfusate component during the procurement surgery. Five pigs in total underwent the experiments, and the surgical information and NMP data are reported in this article.

We present the following article in accordance with the ARRIVE reporting checklist (available at https://dx.doi. org/10.21037/atm-21-1308).

\section{Methods}

In this research, the main objective was to test the feasibility of en bloc procured AMOB for ex situ NMP, avoiding initial cold preservation. The performance during the ex situ NMP process was used to assess the success of en bloc procurement surgery. Five pigs underwent en bloc procurement surgery, and then the AMOBs were transferred to NMP. The poor performance of NMP in cases 1 and 2 was due to repeated hypotension during the procurement surgery. After improving and optimizing the procurement processes, in cases 3 to 5, the NMP process was smooth.

\section{Animals}

In this research, $\mathrm{AMOB}$ were procured from $\mathrm{Ba}-\mathrm{Ma}$ 

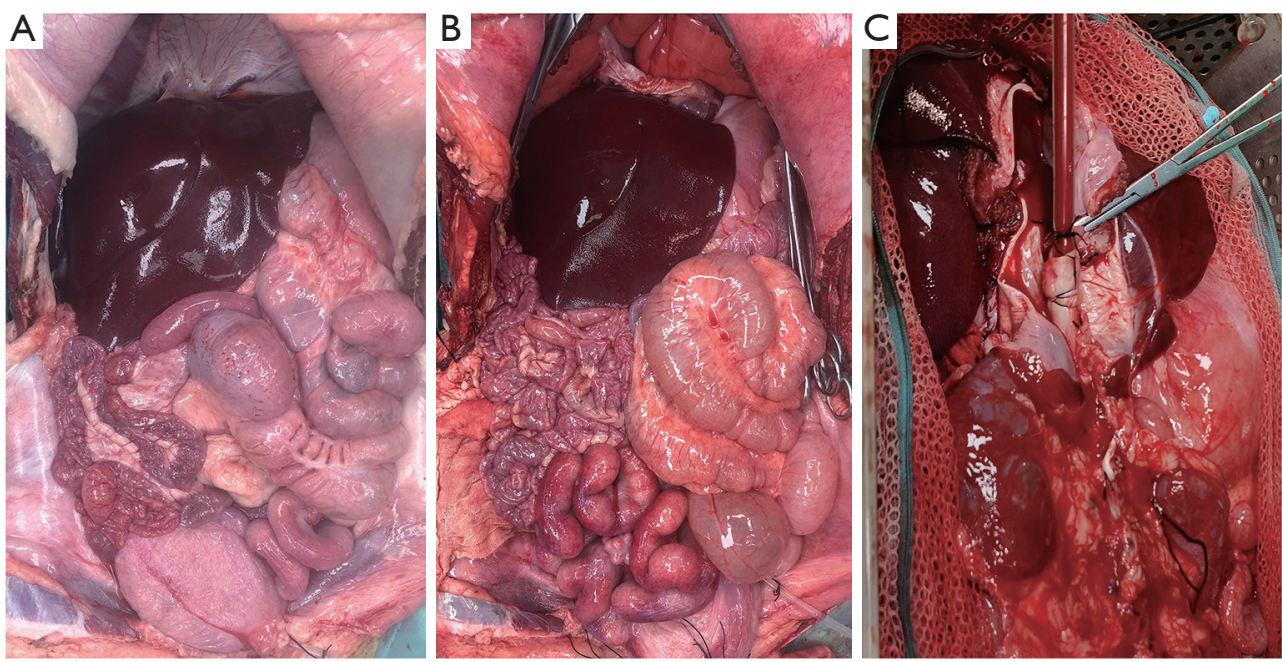

Figure 1 The process of en bloc procurement surgery. (A) showed the visual appearance of the abdominal multiple-organ after open surgery. (B) represent the visual appearance of the abdominal multiple-organ after completing the first and second steps of the procurement surgery (there was only remained the vessel system). (C) showed the abdominal multiple organ block (AMOB) transfer to normothermic machine perfusion (NMP) device to reconstruct blood flow. The dorsal side of the AMOB was kept facing upward during the connection process.

miniature pigs; the median weight of the pigs was 50.0 (43.8-50.0) kg, and the median age was 13 [12-14] months old. There were 2 male and 3 female pigs. The pigs were kept in a comfortable environment with a constant temperature and food and water available ad libitum. The rights of the animals were guaranteed fully according to the Chinese guidelines and the international guidelines (22). Before the operation, all the experimental pigs were banned from food for $12 \mathrm{~h}$ and from water for $4 \mathrm{~h}$. The experimental procedures and protocols were approved by the Committee on Ethics of Animal Experiments of Silver Snake (Guangzhou) Medical (No.: QR-MR003-002-A0), in compliance with Chinese national guidelines for the care and use of animals.

\section{Anesthesia}

All the pigs underwent general anesthesia. In the induction phase, tiletamine/zolazepam ( $5 \mathrm{mg} / \mathrm{kg}$; Virbac, France) was used via intramuscular injection. Endotracheal intubation was executed via laryngoscopy after the onset of anesthesia, which was then connected to a ventilator to maintain breathing during procurement. In the maintenance phase, isoflurane inhalation and intravenous propofol $(10 \mathrm{mg} / \mathrm{kg} / \mathrm{h}$ AstraZeneca, British) were continued throughout the procurement surgery. Meloxicam injection $(0.3 \mathrm{mg} / \mathrm{kg}$; Boehringer Ingelheim, Germany) was used as an analgesic.
The heart rate, electrocardiogram and saturation of blood oxygen were monitored during procurement. Invasive arterial blood pressure monitoring in the right femoral artery and right femoral vein catheterization were used for rapid fluid infusion to maintain circulatory stability. Another venous infusion access was the right ear vein. All operations in this research complied with the principle of minimizing suffering.

\section{En bloc procurement of AMOBs}

The en bloc procurement operation was performed under a heart-beating status. The pig was laid on the operating bed in a supine position. After skin preparation and draping, a midline abdominal incision was made from the subxiphoid to the superior margin of the pubic symphysis, and a transverse incision was made through the umbilicus and ended at the bilateral midaxillary line. An abdominal surgery retractor was used to expose the field of surgery. The en bloc procurement method was applied to remove the AMOB with a normal anatomical relationship. There were three main steps to freeing the AMOB (Figure 1).

First step: free the inferior vena cava (IVC) and aorta artery (AA) from the posterior peritoneum

To open the posterior peritoneum along the right renal lateral border, the dorsal part was dissected to free the 
kidney. Then, the posterior peritoneum was opened along the right side of the IVC, and the IVC and AA were dissected, carefully separating and ligating branch vessels (principally lumbar arteries and veins) sprouting from the $\mathrm{AA}$ and IVC. Isolation was continued upward to the vena cava foramen of the diaphragm and downward to the right common iliac vessels. The same operation was performed on the left side, opening the posterior peritoneum along the left renal lateral border and freeing the kidney. Then, the posterior peritoneum was opened along the left side of the AA upward to the aorta hiatus and downward to the left common iliac vessels to free the AA and IVC absolutely.

\section{Second step: transecting the AMOB from the chest and pelvic cavity except vessels.}

The rectum was dissected carefully and transected close to the anal canal, and the urethra was cut near the bladder. Starting from the inferior pole of the bilateral kidneys, together with the posterior peritoneum, the bilateral ureters were completely dissected to the bladder inlet, preserving the whole bladder and ureter. When the gilt was procured, the uterus and ovaries were preserved, ligated and transected the vagina close to the uterus. After that, the diaphragm was opened and dissected to the bilateral crura of the diaphragm, and the phrenic artery and phrenic vein were dissected and ligated. To isolate the esophagus, it was ligated and transected above the diaphragm.

\section{Last step: clamping and transecting the vessels and moving the AMOB to the NMP device}

Unfractionated heparin was injected via the venous catheter at a dosage of 37,500 U. After waiting approximately 2 minutes, bilateral common iliac vessels were ligated and cut, and then, to clamp and transect the aorta above the diaphragm level, the suprahepatic vena cava was clamped and transected close to the liver. The AMOB was then free and transferred to the NMP device.

The hypotension during procurement in this research refers to the mean arterial pressure (MAP) was less than $60 \mathrm{mmHg}$. Warm ischemia time (WIT) contained the time of MAP below $60 \mathrm{mmHg}$ intraoperative plus the time from donor blockade blood flow to NMP cannulation to rebuild circulation.

\section{Autologous whole blood collection}

Autologous whole blood was the main perfusate component in the initial stage; therefore, blood was collected during the intraoperative period. After opening the abdominal cavity, the left external iliac artery was located, dissected and catheterized to collect blood. The blood was preserved in a blood bag with citrate anticoagulation. It was important to observe the changes in vital signs during exsanguination. A rapid infusion was performed if the blood pressure continuously declined, and exsanguination was suspended when the systolic pressure was lower than $70 \mathrm{mmHg}$.

\section{NMP device and perfusion process}

The NMP device was designed as an open perfusion circuit that provides continuous (no pulsatile) arterial flow at a temperature of $37.5^{\circ} \mathrm{C}$. AMOB is en bloc procurement with the AA and IVC; therefore, the NMP device was designed with only one channel to perfuse the AMOB via the AA. The main components of the NMP device consist of a thermostatic water box, an organ chamber, an opened blood reservoir, a centrifugal pump, a roller pump, an oxygenator and a heat exchanger. Perfusion parameters were monitored in real time by a temperature probe, flow probe and pressure probe (details shown in Figure 2).

The machine was circulating in advance with autologous whole blood, and after the machine was circulating steadily, the organ connection process was executed. The AMOB was moved to the NMP device, and the dorsal side of the AMOB was kept facing upward during the connection process. In the initial phase, the rotation speed of the centrifugal pump was increased, and the pressure was gradually increased. The control system was switched to the constant-pressure model when the pressure value was over $45 \mathrm{mmHg}$. The posterior wall of the AA was carefully examined for points of bleeding, which were ligated, and then the organs were rolled over to the normal anatomic position. The pressure target level was raised to $60-65 \mathrm{mmHg}$, and the flow rate was maintained over $1,000 \mathrm{~mL} / \mathrm{min}$.

\section{Specimens preservation}

Arterial blood gas analysis was executed every $10 \mathrm{~min}$ in the first $30 \mathrm{~min}$ and every hour in the next time. A perfusate specimen was collected each hour to test the liver and kidney function. A $16 \mathrm{~F}$ urinary catheter is inserted into the bladder through the urethral break and the catheter is connected to a negative pressure drainage bottle to drain the urine. The drainage bottle is scaled so that the amount of urine drained can be measured directly. Bile was collected by intubation of the bile duct. The common bile duct was 


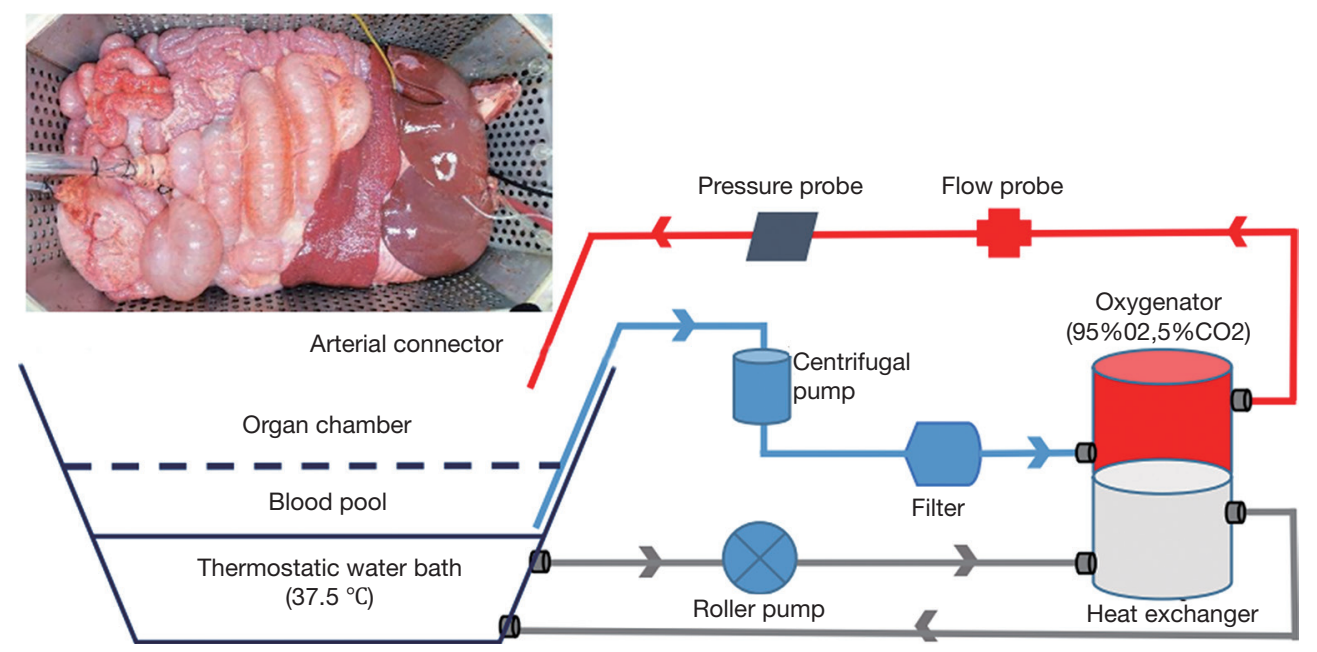

Figure 2 The schematic of normothermic machine perfusion (NMP) device. NMP device was designed as an opened perfusion circuit with continuous (no pulsatile) arterial flow at a temperature of $37.5^{\circ} \mathrm{C}$. The device consists of three layers: from the bottom to surface are the thermostatic water box, the open blood reservoir and the organ chamber respectively. The key components are an oxygenator, heat exchanger, flow probe, pressure probe, centrifugal pump, roller pump and Connecting Pipes.

Table 1 The components of perfusate solution

\begin{tabular}{lc}
\hline Components & Dosage \\
\hline Autologous whole blood & $1,000 \mathrm{~mL}$ \\
Hydroxyethyl starch $130 / 0.4$ and & $1,000 \mathrm{~mL}$ \\
sodium chloride injection & \\
$20 \%$ albumin & $150 \mathrm{~mL}$ \\
$0.9 \%$ sodium chloride injection & $500 \mathrm{~mL}$ \\
Methylprednisolone & $500 \mathrm{mg}$ \\
Heparin & $37,500 \mathrm{U}$ \\
Compound amino acid injection & $250 \mathrm{~mL}$ \\
$10 \%$ calcium gluconate & $20 \mathrm{~mL}$ \\
Metronidazole & $1 \mathrm{~g}$ \\
Cefoperazone sodium and sulbactam sodium & $3 \mathrm{~g}$ \\
\hline
\end{tabular}

located near the pylorus duodenum, and the distal end of the common bile duct was ligated with a \#0 silk suture near the duodenum. A small incision was cut in the common bile duct and bile leakage was seen. The collection of bile was performed using a $2 \mathrm{~mm}$ diameter micropump extension tube inserted from the incision into the common bile duct up to the level of the common hepatic duct and secured with a \#0 silk suture. The cystic duct was separated near the neck of the gallbladder and ligated with a \#0 silk suture.

\section{Statistical methods}

This research is mainly an observational study. There were 5 cases in total, and the measurement data are expressed as the median and the range.

\section{Results \\ Perfusate elements}

Whole blood was the main element in the perfusate; hetastarch, 20\% albumin, Sodium chloride injection, Compound Amino Acid Injection and methylprednisolone were added, the total volume of perfusate was approximate to $3,000 \mathrm{~mL}$ at first. Ten percent calcium gluconate was used for regulating blood calcium concentration, and 5\% sodium bicarbonate was used to adjust the $\mathrm{pH}$. Metronidazole and cefoperazone sodium and sulbactam sodium were used to prevent infection. Autologous whole blood was used in the beginning, and allogeneic whole blood was used as a standby liquid to supplement the perfusate if the cross-match test was negative (the detailed information showed in Table 1).

\section{En bloc procurement surgery}

Five Ba-Ma miniature pigs underwent en bloc procurement surgery to acquire the AMOB for NMP. The pigs were 2 males and 3 females, their median weight and median 
Table 2 Basic information of en bloc procurement surgery

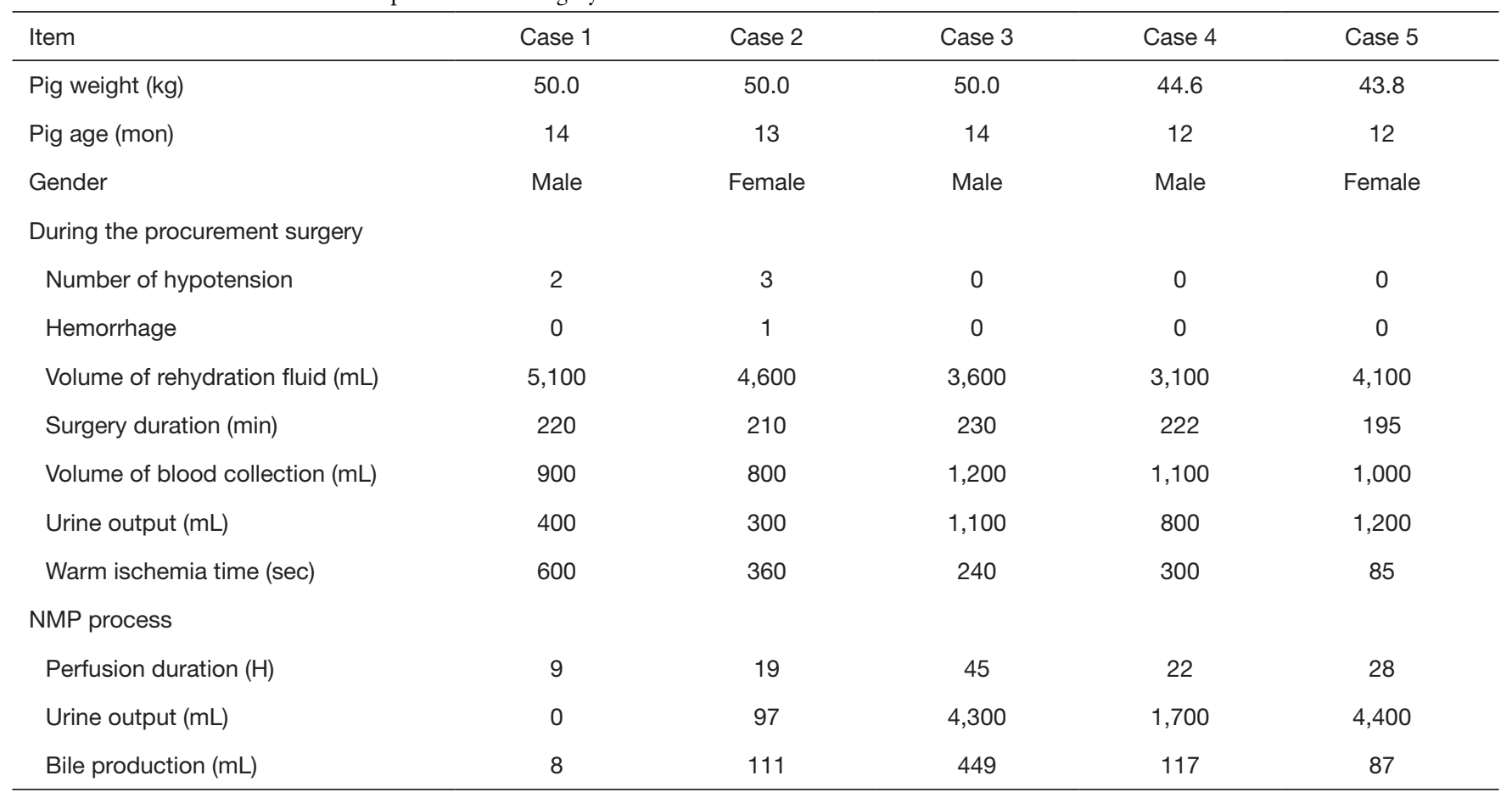

Hypotension: refers to the mean arterial pressure (MAP) was less than $60 \mathrm{mmHg}$; warm ischemia time: the time of MAP below $60 \mathrm{mmHg}$ intraoperative plus the time from donor blockade blood flow to normothermic machine perfusion (NMP) cannulation to rebuild circulation.

age were $50.0(43.8-50.0) \mathrm{kg}$ and $13[12-14]$ months respectively. The median time of procurement surgery was 220 [195-230] min, and the median warm ischemia time (WIT) was 240 [85-600] sec.

In the first attempts at this surgery, case 1 and case 2 suffered from repeated hypotension, and case 2 exhibited hemorrhage. AMOB functions were damaged in both cases due to warm ischemia injury caused by hypotension. After improved and optimized procurement processes, hypotension and hemorrhage were prevented in the remaining cases. The vital signs of cases 3 to 5 remained stable during the whole procurement, and the volume of fluid supply during the procurement was less than that in cases 1 and 2 (Table 2). Meanwhile, the volume of blood collection and urine output were greater than those in cases 1 and 2 during the procurement. The WIT gradually declined and was only 85 seconds in case 5 (Table 2).

During the NMP process, the perfusion duration of case 3 to case 5 was significantly longer than that of case 1 and case 2, and the longest ex situ maintenance time reached was $45 \mathrm{~h}$. The urine output during the NMP was also obviously higher in cases 3 to 5 , the median volume of urine was 4,300 $[1,700-4,400] \mathrm{mL}$. However, case 1 was urine absent, and case 2 only had $97 \mathrm{~mL}$ of urine. The bile produced in case 1 during ex vivo NMP was only $8 \mathrm{~mL}$ but was normal in case 2 at a volume of $111 \mathrm{~mL}$ (Table 2).

\section{Reperfusion and perfusion parameters}

A constant pressure mode was adopted during the NMP in this research, and the target value of pressure was set between 60 and $70 \mathrm{mmHg}$. A pressure limited to $70 \mathrm{mmHg}$ was used to avoid hemorrhage of the intestinal mucosa, which has been observed in many failed attempts. The vasodilator papaverine was continuously infused $(6-12 \mathrm{mg} / \mathrm{h})$ to maintain an appropriate flow rate during NMP, and the target value of the flow rate was kept above $1,000 \mathrm{~mL} / \mathrm{min}$.

A smooth reperfusion process indicates the success of en bloc procurement surgery. The organ functions of cases 1 and 2 were damaged due to hypotension during en bloc procurement. Therefore, the flow rate was increased slowly in the beginning and could not sustain stability even when continuously infused with a high dose of papaverine $(48 \mathrm{mg} / \mathrm{h})$. The maximum flow rate was less than $1,000 \mathrm{~mL} / \mathrm{min}$ in case 1 and case 2 (Figure $3 A$ ). By contrast, at the same constant pressure mode with 60 to $65 \mathrm{mmHg}$, 

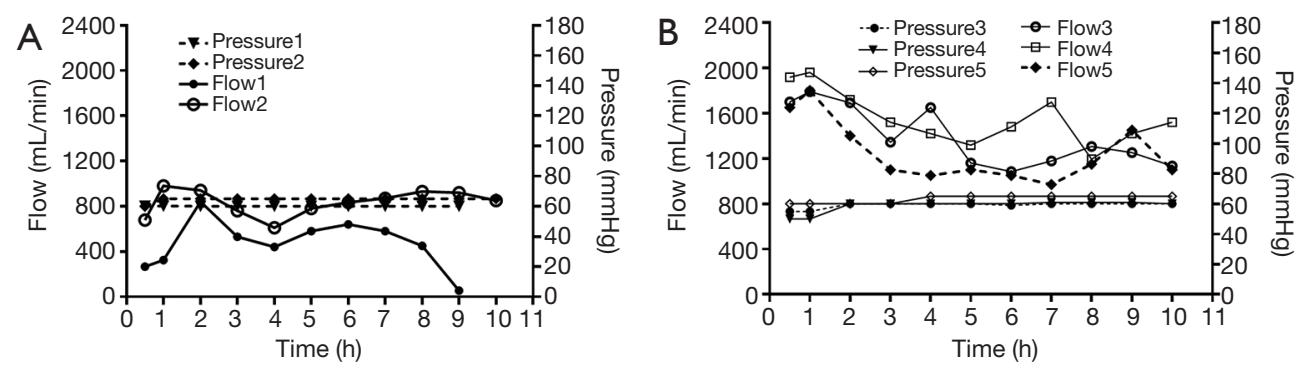

Figure 3 Variation curves of the flow and pressure in the first $10 \mathrm{~h}$ normothermic machine perfusion (NMP). (A) represent the changes of perfusion parameters in cases 1 and 2 . The flow rate was slow upturn in the beginning, and the maximum flow rate was less than $1,000 \mathrm{~mL} / \mathrm{min}$. Brief increase in flow rate in case 1 followed by a rapid decrease. Contrary to that, at the same constant pressure mode with 60 to $65 \mathrm{mmHg}$, (B) showed the flow rate in cases 3 to 5 increased rapidly at first, and the value even exceed $1,500 \mathrm{~mL} / \mathrm{min}$. In the first $10 \mathrm{~h}$ NMP, the flow rate consistently exceeding $1,000 \mathrm{~mL} / \mathrm{min}$.

the flow rate in cases 3 to 5 increased rapidly, and the value exceeded $1,500 \mathrm{~mL} / \mathrm{min}$ in the first phase. In the first $10 \mathrm{~h}$ of NMP, the flow rate was maintained at over $1,000 \mathrm{~mL} / \mathrm{min}$ (Figure 3B).

A good visual appearance of the AMOB and active gastrointestinal peristalsis after restored blood flow with NMP also indicate the success of en bloc procurement surgery. The liver, spleen and intestines showed uniform perfusion and a scarlet appearance in the first hour in cases 3 to 5 (Figure 4), and as time passed, the gross appearance of the organs gradually became gray due to anemia, but gastrointestinal peristalsis continuously existed and was active. Fewer secretions accumulated in the intestinal lumen, and no obvious intestinal mucosal bleeding was observed (Figure 4, 3 and 7 h). In case 1 and case 2, the organs also received uniform perfusion in the beginning, but at 3 and $7 \mathrm{~h}$, the intestine became swollen and appeared black, indicating intestinal mucosal bleeding. The flow rate continuously declined, the organ gross appearance became black, and gastrointestinal peristalsis was lost. NMP was maintained for only $9 \mathrm{~h}$ in case 1 , and perfusion was sustained in case 2 with continuous infusion of a high dose of papaverine $(48 \mathrm{mg} / \mathrm{h})$.

\section{Lactic acid levels}

The oxygen flow was set at $2 \mathrm{~L} / \mathrm{min}$, and the oxygen concentration was $95 \%$ in the beginning. The changes in lactic acid levels reflected tissue perfusion and cell metabolism. In case 1 , WIT reached $10 \mathrm{~min}$ due to repeated hypotension during the procurement surgery, the lactic acid level was over $12 \mathrm{mmol} / \mathrm{L}$ at first, and the value rapidly increased exceeding $20 \mathrm{mmol} / \mathrm{L}$ at $1 \mathrm{~h}$ due to the low flow during NMP. The value of lactic acid in case 2 was obviously lower than that in case 1 in the beginning, but given the poor perfusion parameters due to organ injury in the procurement, the level of lactic acid increased rapidly and exceeded $20 \mathrm{mmol} / \mathrm{L}$ at $6 \mathrm{~h}$. The level of lactic acid in case 3 was slightly high at first, but the value remained stable in the first $10 \mathrm{~h}$. The levels of lactic acid in cases 4 and 5 were low and remained stable during the first $10 \mathrm{~h}$, which indicated that good organ function was preserved and adequate blood perfusion was achieved (Figure 5).

\section{Organ function}

Perfusate samples were regularly collected to detect liver and kidney function during NMP. The results showed that the alanine aminotransferase (ALT) level and the aspartate aminotransferase (AST) level were higher in cases 1 and 2 than in cases 3 to 5. The levels of ALT and AST rapidly increased in the later period in cases 1 and 2 but remained stable in cases 3 to 5 (Figure $6 A, B$ ). The creatine levels all remained stable in the first $10 \mathrm{~h}$ of NMP, but the volume of urine in case 1 and case 2 was significantly less than that in cases 3 to 5 in this period (Figure 6C). The changes in glucose levels in all cases proved that the organs recovered metabolism (Figure 6D).

\section{Pathological results}

Pathological specimens of liver and small intestines were reserved at a regular interval. Unfortunately, no pathological specimens were kept in case 1 . In cases 2 to 5 , the retained small intestine and liver specimens were paraffin-embedded and sectioned, and then hematoxylin-eosin staining was 

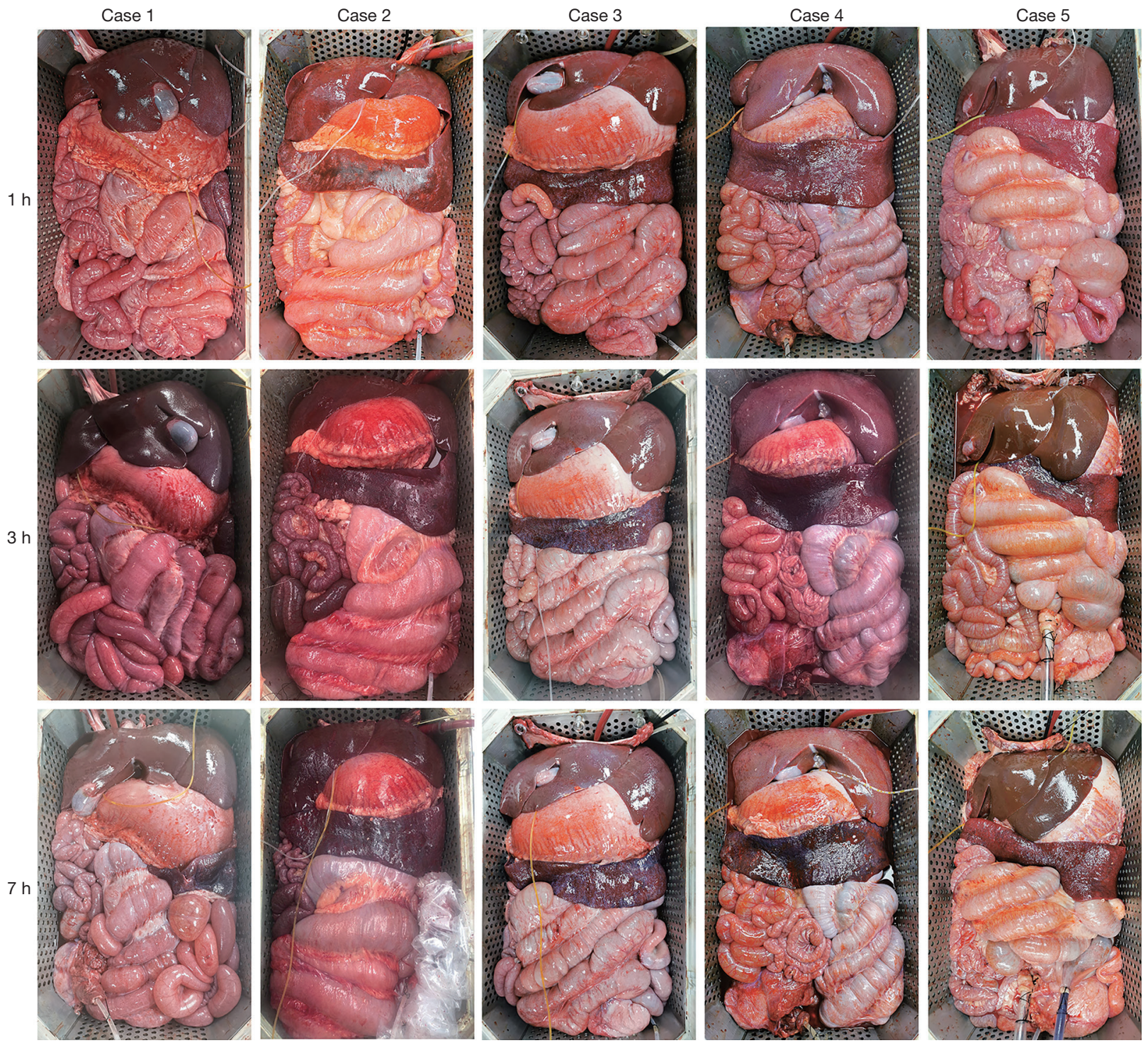

Figure 4 Visual appearance of abdominal multiple organ block (AMOB) during the ex vivo normothermic machine perfusion (NMP). Visual appearance at 1, 3 and $7 \mathrm{~h}$ after NMP in all 5 cases. At $1 \mathrm{~h}$, the organs also received uniform perfusion in case 1 and case 2, but after perfusion at 3 and $7 \mathrm{~h}$, the intestine became swollen and black appearance, which indicates bleeding in the intestinal mucosal. In case 2, perfusion was sustained with continuous infusion of a high dose of papaverine ( $48 \mathrm{mg} / \mathrm{h})$. In cases 3 to 5 , the liver, spleen and intestines showed uniform perfusion and a scarlet appearance at $1 \mathrm{~h}$. The visual appearance of the organs gradually became gray due to anemia at 3 and $7 \mathrm{~h}$, but gastrointestinal peristalsis continuously existed and was active. Fewer secretions accumulated in the intestinal lumen, and no obvious intestinal mucosal bleeding was observed.

performed. The pathological results of cases 2 to 5 were showed in Figure 7 ( $\times 200$ magnification, the scale length is $100 \mu \mathrm{m})$.

In case 2, Pathology showed the villi of the small intestine was preserved well at $1 \mathrm{~h}$, but at 6 and $12 \mathrm{~h}$, the intestinal pathology showed significant damage and the villi structure was obviously damaged and disintegrating. The structure of the liver in case 2 was preserved well at $1 \mathrm{~h}$, and liver pathology at 6 and $12 \mathrm{~h}$ also did not show significant damage. The portal area kept intact, the hepatic cell was integrated and showed mild swelling, and the hepatic sinusoid structure was general normal. In cases 3 , the small 

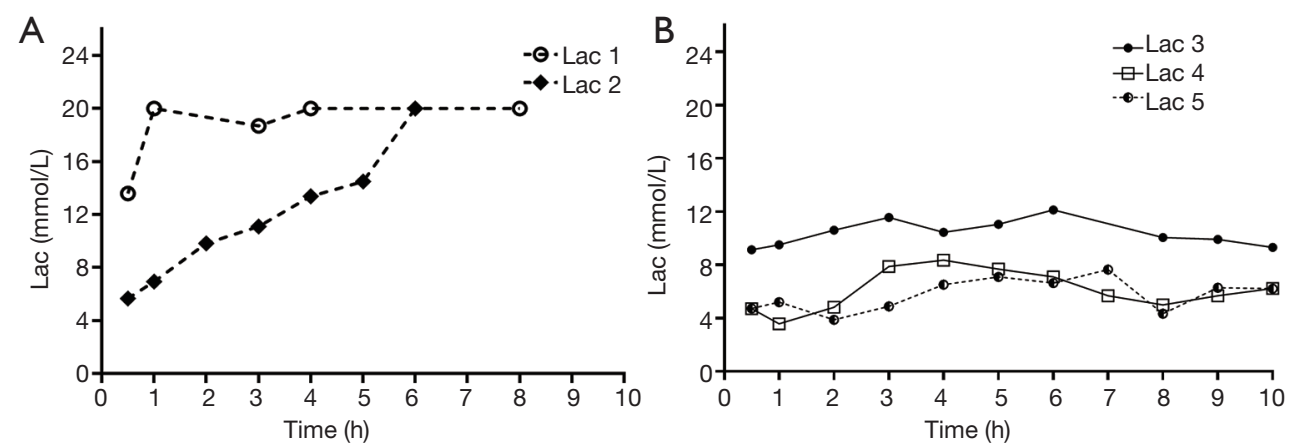

Figure 5 Change curve of lactic acid level in the first 10 h normothermic machine perfusion (NMP). (A) showed the lactic acid level was over $12 \mathrm{mmol} / \mathrm{L}$ in case 1 at first, and rapidly increased exceed $20 \mathrm{mmol} / \mathrm{L}$ at $1 \mathrm{~h}$. In case 2, the lactic acid level was obviously lower than that in case 1 in the beginning, but the value continued to rise, exceeding $20 \mathrm{mmol} / \mathrm{L}$ at $6 \mathrm{~h}$ after NMP. (B) The lactic acid level in case 3 was slightly high at first, but the value remained stable in the first $10 \mathrm{~h}$ NMP. The lactic acid level in cases 4 and 5 were low and remained stable during the first $10 \mathrm{~h}$, which indicated well maintained organ function during the procurement and adequate blood flow during the NMP.
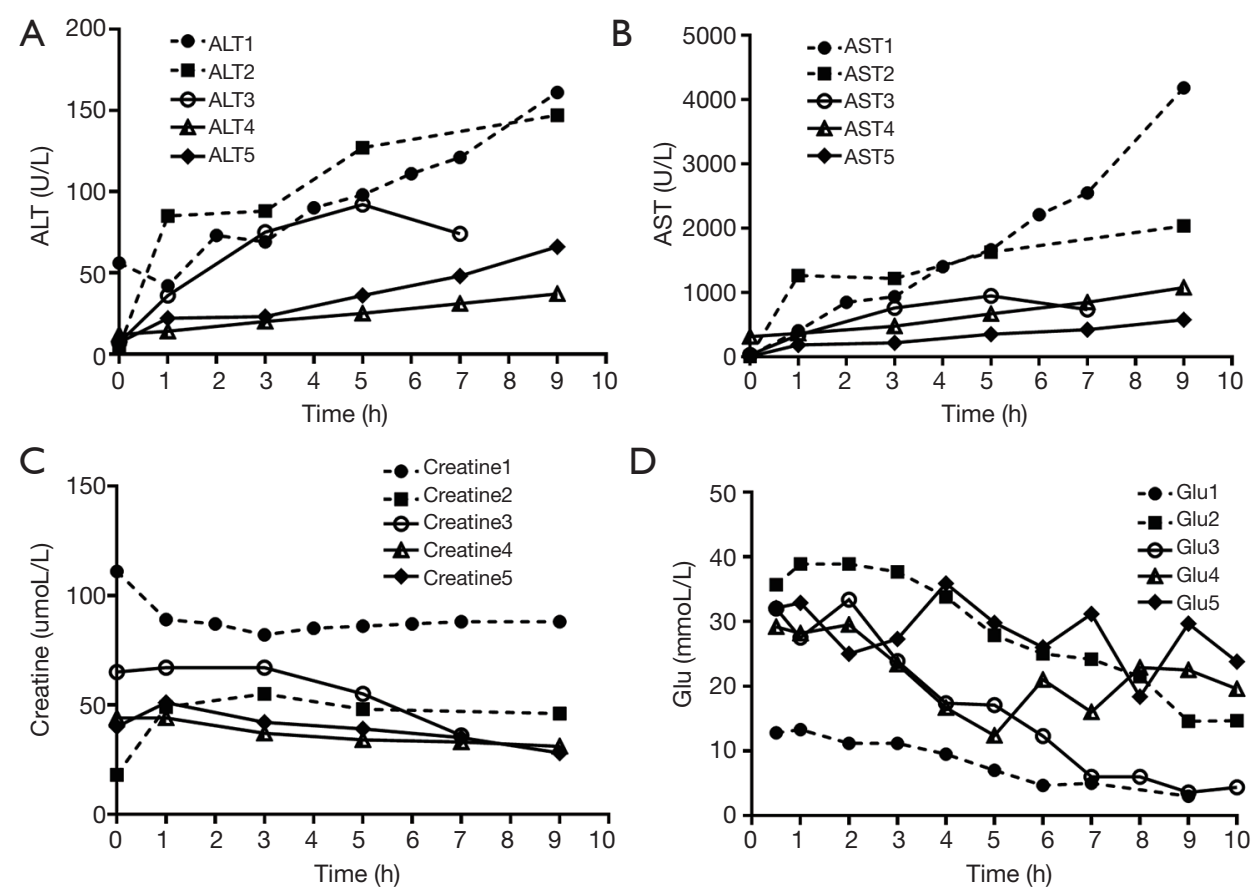

Figure 6 Variation curves of liver and kidney function and glucose levels in the first $10 \mathrm{~h}$ normothermic machine perfusion (NMP). (A and B) showed the alanine aminotransferase (ALT) and aspartate aminotransferase (AST) levels were higher in cases 1 and 2 than in cases 3 to 5. The ALT and AST levels continuously elevated in cases 1 and 2 in first $10 \mathrm{~h}$, but slightly elevated in cases 3 to 5 . (C) showed the creatine levels in all cases remained stable in the first $10 \mathrm{~h}$, but the urine output in cases 1 and 2 were significantly less than that in cases 3 to 5 in this period. The changes of glucose level in (D) indicate that the organs underwent metabolism in all cases. Cases 3 to 5 irregular addition of glucose, but cases 1 and 2 without any added glucose during NMP. 

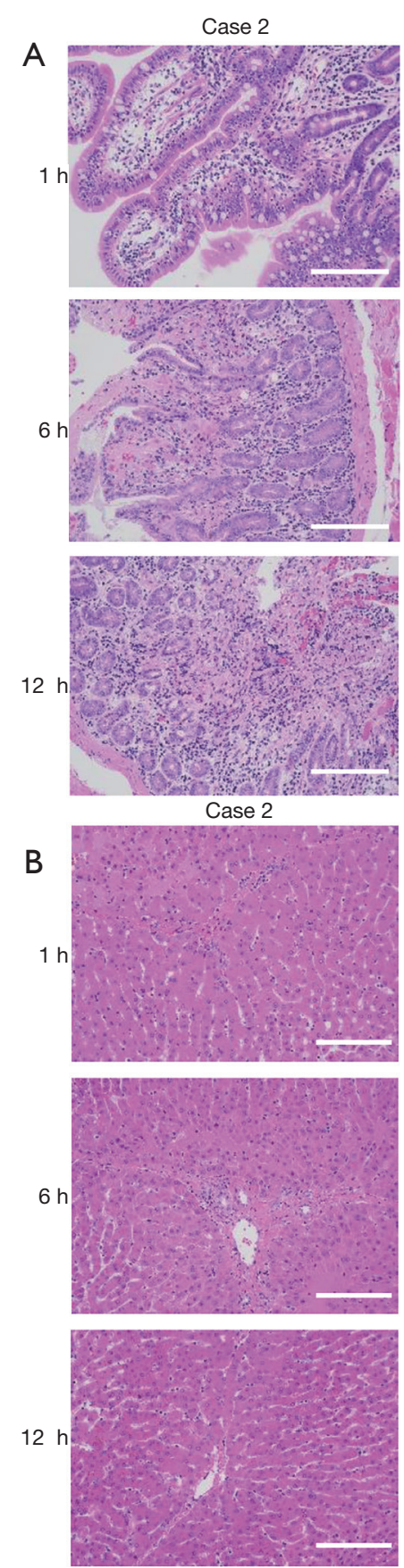
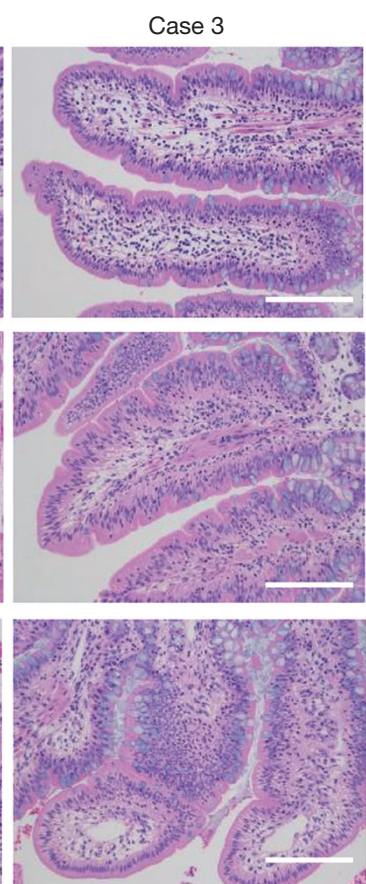

Case 3
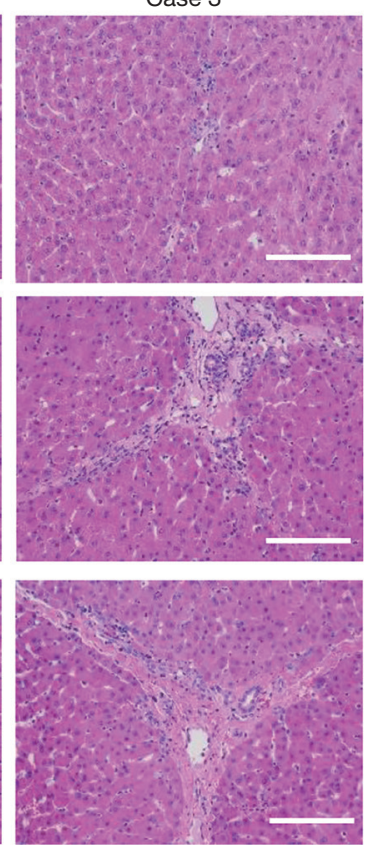
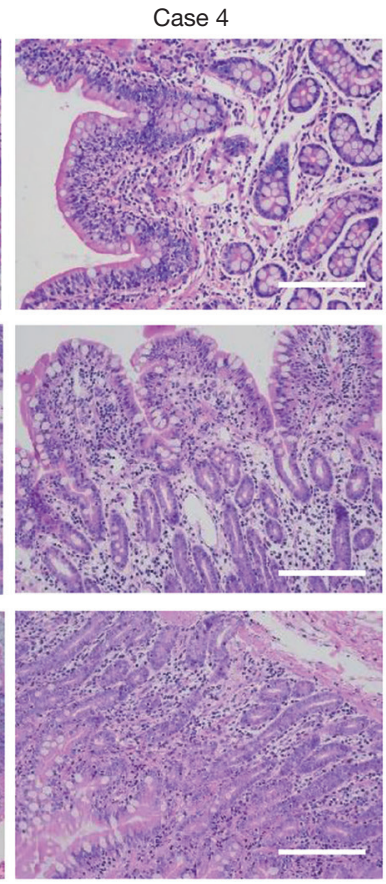

Case 4
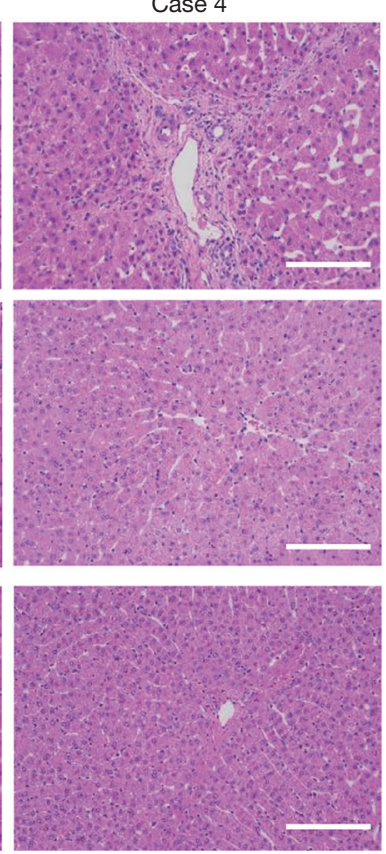
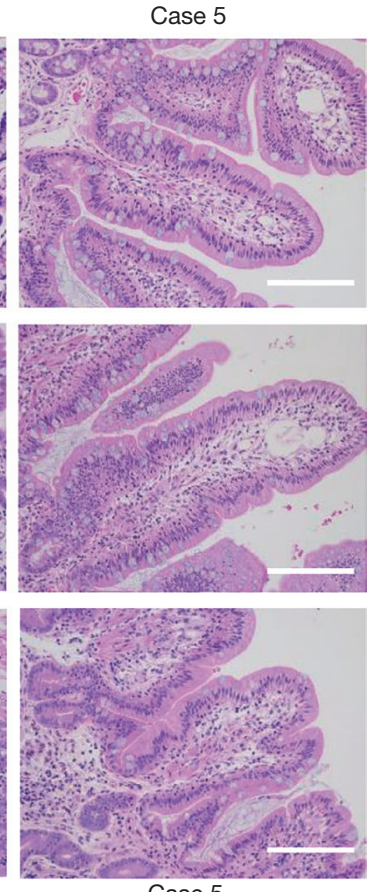

Case 5
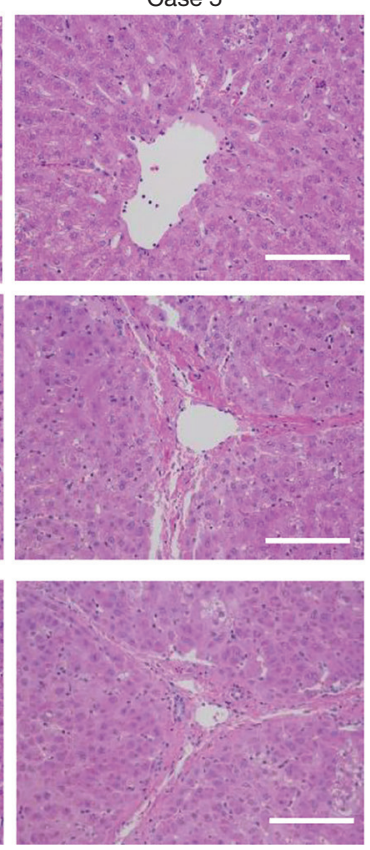

Figure 7 Pathological changes of liver and small intestines during the normothermic machine perfusion (NMP). This figure showed pathological changes of small intestines (A) and liver (B), hematoxylin and eosin (HE) staining with 200x magnification, the scale length is $100 \mu \mathrm{m}$. In case 2, Pathology showed the villi of the small intestine was preserved well at $1 \mathrm{~h}$, but at 6 and $12 \mathrm{~h}$, the intestinal pathology showed significant damage and the villi structure was obviously damaged and disintegrating. The structure of the liver in case 2 was preserved well at $1 \mathrm{~h}$, and liver pathology at 6 and $12 \mathrm{~h}$ also did not show significant damage. The portal area kept intact, the hepatic cell was integrated and showed mild swelling, and the hepatic sinusoid structure was general normal. In cases 3 , the small intestine pathology showed the villi of the small intestine were preserved well at 1,6 and $12 \mathrm{~h}$, the small intestines pathology in cases 4 and 5 were also kept well. During the same period, no obvious changes in liver pathology were observed in cases 3 to 5 . The portal area kept intact, the hepatic cells were integrated and showed mild swelling, and the hepatic sinusoid structure were also general normal. 
intestine pathology showed the villi of the small intestine were preserved well at 1, 6 and $12 \mathrm{~h}$, the small intestines pathology in cases 4 and 5 were also kept well. During the same period, no obvious changes in liver pathology were observed in cases 3 to 5 . The portal area kept intact, the hepatic cells were integrated and showed mild swelling, and the hepatic sinusoid structure were also general normal.

\section{Discussion}

This article describes the first attempt to en bloc procure AMOB for NMP. The results confirmed that it was feasible to obtain AMOB for NMP through en bloc procurement surgery, and only a reliable procurement surgery could achieve a good NMP effect. In the first 2 cases, due to insufficient skill in intraoperative coordination and inadequate knowledge of local anatomy, repeated hypotension occurred during the surgery, the function of the $\mathrm{AMOB}$ was damaged, and the perfusion performance was inadequate during the NMP period. After improving the procurement process and optimizing the details, the function of the $\mathrm{AMOB}$ was preserved well in the remaining 3 cases, and the NMP process was smooth.

Interestingly, although the WIT was only 600 and 360 seconds in cases 1 and 2, respectively, the multipleorgan function suffered severe damage and made it difficult to maintain the NMP. In contrast, in the previous porcine DCD model, the WIT was as long as 30 to $60 \mathrm{~min}$, and the NMP effect was still better $(15,23)$. Possible reasons to consider include that although the recorded WIT was not long, local tissue ischemia persisted during procurement surgery, especially in the intestinal tissues. The volume of the gastrointestinal tract was large, and repeated hypotension and exposure of the surgical field may persistently impact the intestinal blood supply, causing severe impairment of organ function and thus leading to poor NMP perfusion. In addition, the intraoperative urine output was significantly lower in cases 1 and 2 than in cases 3 to 5 (Table 2), which also indicates circulatory instability.

An improvement measure was eliminated from the process of cold preservation, which is a routine operation in the clinic and in experiments $(24,25)$. Considering the good mobility of the NMP device and the expected short duration of WIT, to save costs and simplify the operation steps, the process of cold preservation was eliminated in the experimental design. To shorten the time of organ ischemia, the blood vessels were dissociated at the end of procurement surgery, and the NMP device was also operated in advance before the vessels were dissociated. In the fifth experiment, the time from blocking blood perfusion in the donor to transfer to the NMP device to re-establish blood flow was only 85 seconds.

The NMP process was successful, demonstrating the feasibility of this cold-free preservation procedure. In addition, if cold preservation is carried out, the АМОВ will undergo a process of cooling and rewarming in a short time, and whether rapid cooling and rewarming affect the NMP results is another problem. However, one study demonstrated that avoiding cold preservation does not improve liver quality in a porcine DCD model of NMP (26). Actually, eliminating cold preservation also resulted in some slight negative effects; most visible was that the lactic acid level was slightly higher in the beginning. Because there was no cold flushing, a large amount of autologous blood and metabolic waste accumulated in the vascular lumen and circulated into the perfusate after NMP operation. However, the negative effects were limited, and the lactic acid level remained stable in the first $10 \mathrm{~h}$ and even decreased to normal upon further perfusion.

Another improvement measure was autologous whole blood as an NMP perfusate in the initial phase. Previous animal experiments by our team found that cross-use of allogeneic pig blood may cause hemolysis and lead to perfusion failure for the complex blood group system in pigs (27). Therefore, to reduce the occurrence of this risk and improve the outcome of the NMP experiment, autologous whole blood was used as the component of perfusate at the beginning, and allogeneic pig blood from the slaughterhouse was used as a supplementary liquid after the cross-matching test was negative. This improvement process could reduce the number of experimental pigs, decrease experimental costs, and be more in line with animal ethical requirements.

There were more challenges in this research than in the few experiments reported on the use of autologous blood (28). The procurement surgery was performed under the heart-beating status in our research, and due to abandonment of cold preservation, the collection of whole blood must be accomplished during the surgery to advance the operation of the NMP device. Circulatory instability was observed during blood collection in the first 2 cases of the experiment, leading to organ function damage and poor NMP performance. The lactic acid level rapidly increased in a short period. After the modified and optimized process, the en bloc procurement process of the following 3 cases was very smooth. There was no obvious hypotension in the blood 
collection process, and organ function was maintained well. In total, 1,000-1,200 $\mathrm{mL}$ whole blood was collected during the procurement surgery, basically meeting the needs of the initial stage perfusion.

There are also some limitations to this research. First, this is just an observational study to prove the feasibility of en bloc procured AMOB for NMP without initial cold preservation. More accurate detection indicators or transplant models are needed to confirm the organ functions of AMOBs. Second, although AMOBs were ex situ maintained with NMP for a relatively long period of time, organ function was not optimally maintained, and the optimal perfusion strategy needs to be explored. Third, AMOBs were procured from heart-beating pigs in this research, and AMOBs procured from donation after cardiac death (DCD) mode may be more meaningful.

To our knowledge, this is the first report of relevant research. We proved the feasibility of en bloc procurement of AMOB to ex situ NMP without initial cold preservation. This is a meaningful attempt, and according to the concept of organ-oriented research and treatment, we can use this model for scientific research and teaching, such as drug development, organ-to-organ interactions, laparoscopic live organ training, etc. If we can use the discarded abdominal organs of pigs in slaughterhouses to build models, it will greatly reduce the cost and be more in line with animal ethics, which is the next direction of our research.

\section{Conclusions}

In conclusion, procuring $\mathrm{AMOBs}$ from heart-beating pigs with en bloc procurement surgery for NMP without cold preservation is feasible. Autologous whole blood as a perfusate component in the beginning of NMP is a feasible measure, and approximately $1,000 \mathrm{~mL}$ of whole blood collected during procurement surgery had a stable circular status.

\section{Acknowledgments}

Funding: This work was supported by the National Natural Science Foundation of China (81401324 and 81770410), Guangdong Basic and Applied Basic Research Foundation (2020A1515011557, 2020A1515010903), Science and Technology Planning Project of Guangdong Province (2016A020215048), Guangdong Provincial Key Laboratory of Organ Donation and Transplant Immunology, The First Affiliated Hospital, Sun Yat-sen University, Guangzhou, China (2020B1212060026), Guangdong Provincial
International Cooperation Base of Science and Technology (Organ Transplantation), The First Affiliated Hospital, Sun Yat-sen University, Guangzhou, China (2015B050501002).

\section{Footnote}

Reporting Checklist: The authors have completed the ARRIVE reporting checklist. Available at https://dx.doi. org/10.21037/atm-21-1308

Data Sharing Statement: Available at https://dx.doi. org/10.21037/atm-21-1308

Peer Review File: Available at https://dx.doi.org/10.21037/ atm-21-1308

Conflicts of Interest: All authors have completed the ICMJE uniform disclosure form (available at https://dx.doi. org/10.21037/atm-21-1308). The authors have no conflicts of interest to declare.

Ethical Statement: The authors are accountable for all aspects of the work in ensuring that questions related to the accuracy or integrity of any part of the work are appropriately investigated and resolved. Experiments were performed under a project license (No.: QR-MR003002-A0) granted by the Committee on Ethics of Animal Experiments of Silver Snake (Guangzhou) Medical, in compliance with Chinese national guidelines for the care and use of animals.

Open Access Statement: This is an Open Access article distributed in accordance with the Creative Commons Attribution-NonCommercial-NoDerivs 4.0 International License (CC BY-NC-ND 4.0), which permits the noncommercial replication and distribution of the article with the strict proviso that no changes or edits are made and the original work is properly cited (including links to both the formal publication through the relevant DOI and the license). See: https://creativecommons.org/licenses/by-nc-nd/4.0/.

\section{References}

1. Jamieson NV, Sundberg R, Lindell S, et al. Preservation of the canine liver for 24-48 hours using simple cold storage with UW solution. Transplantation 1988;46:517-22.

2. Todo S, Nery J, Yanaga K, et al. Extended preservation of human liver grafts with UW solution. JAMA 1989;261:711-4. 
3. Eshmuminov D, Becker D, Bautista Borrego L, et al. An integrated perfusion machine preserves injured human livers for 1 week. Nat Biotechnol 2020;38:189-98.

4. Collins GM, Bravo-Shugarman M, Terasaki PI. Kidney preservation for transportation. Initial perfusion and 30 hours' ice storage. Lancet 1969;2:1219-22.

5. Stewart ZA. UW solution: still the "gold standard" for liver transplantation. Am J Transplant 2015;15:295-6.

6. Moers C, Smits JM, Maathuis MH, et al. Machine perfusion or cold storage in deceased-donor kidney transplantation. N Engl J Med 2009;360:7-19.

7. Kox J, Moers C, Monbaliu D, et al. The Benefits of Hypothermic Machine Preservation and Short Cold Ischemia Times in Deceased Donor Kidneys. Transplantation 2018;102:1344-50.

8. De Deken J, Kocabayoglu P, Moers C. Hypothermic machine perfusion in kidney transplantation. Curr Opin Organ Transplant 2016;21:294-300.

9. Schlegel A, Kron P, Graf R, et al. Warm vs. cold perfusion techniques to rescue rodent liver grafts. J Hepatol 2014;61:1267-75.

10. Schlegel A, Muller X, Kalisvaart M, et al. Outcomes of DCD liver transplantation using organs treated by hypothermic oxygenated perfusion before implantation. J Hepatol 2019;70:50-7.

11. van Rijn R, Schurink IJ, de Vries Y, et al. Hypothermic Machine Perfusion in Liver Transplantation - A Randomized Trial. N Engl J Med 2021;384:1391-401.

12. Nasralla D, Coussios CC, Mergental H, et al. A randomized trial of normothermic preservation in liver transplantation. Nature 2018;557:50-6.

13. Mergental H, Laing RW, Kirkham AJ, et al. Transplantation of discarded livers following viability testing with normothermic machine perfusion. Nat Commun 2020;11:2939.

14. op den Dries S, Karimian N, Sutton ME, et al. Ex vivo normothermic machine perfusion and viability testing of discarded human donor livers. Am J Transplant 2013;13:1327-35.

15. He X, Ji F, Zhang Z, et al. Combined liver-kidney perfusion enhances protective effects of normothermic perfusion on liver grafts from donation after cardiac death. Liver Transpl 2018;24:67-79.

16. Voiglio EJ, Gorry FC, Margonari J, et al. Rat multiple organ blocks: microsurgical technique of removal for ex vivo aerobic organ preservation using a fluorocarbon emulsion. Microsurgery 2000;20:109-15.

17. Chien S, Diana JN, Oeltgen PR, et al. Eighteen to 37 hours' preservation of major organs using a new autoperfusion multiorgan preparation. Ann Thorac Surg 1989;47:860-7.

18. Zhang Z, Tang Y, Zhao Q, et al. Association of Perfusion Characteristics and Posttransplant Liver Function in Ischemia-Free Liver Transplantation. Liver Transpl 2020;26:1441-54.

19. He X, Chen G, Zhu Z, et al. The First Case of IschemiaFree Kidney Transplantation in Humans. Front Med (Lausanne) 2019;6:276.

20. He X, Guo Z, Zhao Q, et al. The first case of ischemiafree organ transplantation in humans: A proof of concept. Am J Transplant 2018;18:737-44.

21. Zhao Q, Nie Y, Guo Z, et al. The future of organ-oriented research and treatment. Hepatobiliary Surg Nutr 2019;8:502-5.

22. Sherwin CM, Christiansen SB, Duncan IJ, et al. Guidelines for the ethical use of animals in applied ethology studies. Applied Animal Behaviour Science 2003;81:291-305.

23. Liu Q, Nassar A, Farias K, et al. Comparing Normothermic Machine Perfusion Preservation With Different Perfusates on Porcine Livers From Donors After Circulatory Death. Am J Transplant 2016;16:794-807.

24. Ghinolfi D, Rreka E, De Tata V, et al. Pilot, Open, Randomized, Prospective Trial for Normothermic Machine Perfusion Evaluation in Liver Transplantation From Older Donors. Liver Transpl 2019;25:436-49.

25. Marecki H, Bozorgzadeh A, Porte RJ, et al. Liver ex situ machine perfusion preservation: A review of the methodology and results of large animal studies and clinical trials. Liver Transpl 2017;23:679-95.

26. Nostedt JJ, Churchill T, Ghosh S, et al. Avoiding initial hypothermia does not improve liver graft quality in a porcine donation after circulatory death (DCD) model of normothermic perfusion. PLoS One 2019;14:e0220786.

27. Smith DM, Newhouse M, Naziruddin B, et al. Blood groups and transfusions in pigs. Xenotransplantation 2006;13:186-94.

28. Minor T, Efferz P, Fox M, et al. Controlled oxygenated rewarming of cold stored liver grafts by thermally graduated machine perfusion prior to reperfusion. Am J Transplant 2013;13:1450-60.

Cite this article as: Chen C, Chen M, Lin X, Guo Y, Ma Y, Chen Z, Ju W, He X. En bloc procurement of porcine abdominal multiple organ block for ex situ normothermic machine perfusion: a technique for avoiding initial cold preservation. Ann Transl Med 2021;9(14):1116. doi: 10.21037/ atm-21-1308 